VOL. $2(1970), 101-106$.

\title{
Quantum logic as an implication algebra
}

\section{P. D. Finch}

For the purpose of this paper a logic is defined to be a non-empty set of propositions which is partially ordered by a relation of logical implication, denoted by "s" , and which, as a poset, is orthocomplemented by a unary operation of negation. The negation of the proposition $x$ is denoted by $N x$ and the least element in the logic is denoted by 0 , we write $N 0=1$. A binary operation " $\rightarrow$ " is introduced into a logic, the operation is interpreted as material implication so that $" x \rightarrow y$ " is a proposition of the logic and is read as " $x$ materially implies $y^{\prime \prime}$. If material implication has the properties

Il. $(x \rightarrow 0)=N x$,

I2. if $x \leq y$ then $(z \rightarrow x) \leq(z \rightarrow y)$,

I3. if $x \leq y$ then $x \rightarrow(y \rightarrow z)=x \rightarrow z$,

I4. $x \leq\{y \rightarrow N(y \rightarrow N x)\}$,

then the logic is an orthomodular lattice. The lattice operations of join and meet are given by

$$
\begin{aligned}
& x \vee y=N x \rightarrow N(N x \rightarrow N y) \\
& x \wedge y=N(x \rightarrow N(x \rightarrow y))
\end{aligned}
$$

and, in terms of the lattice operations, the material implication is given by

$$
(x \rightarrow y)=(y \wedge x) \vee N x .
$$

Moreover the logic is a Boolean algebra if, and only if, in addition to the properties above, material implication satifies

$$
\text { I5. }(x \rightarrow y)=(N y \rightarrow N x) \text {. }
$$

Received 14 October 1969. 


\section{Introduction}

In what follows we adopt, without repetition of them, the definitions and terminology introduced in the summary above. For the connection between the logic of quantum mechanics and orthomodular lattices we refer to Finch [3] and [5].

The statements of the summary are immediate consequences of the results in Finch [4]. Thus suppose that $L$ is a logic which admits a binary relation " $\rightarrow$ " with properties Il through to I4. For each $y$ in $L$ define a map $\pi_{y}$ of $L$ into itself by the equation

$$
x \pi_{y}=N(y+N x)
$$

By I1, $I \pi_{y}=y$ and I2, I3 and I4 just assert that the $\pi_{y}$ form a Sasaki set of projections on $L$. By the theorem of Finch [4] it follows that $L$ is an orthomodular lattice, that

$$
(x+y)=(y \wedge x) \vee N x
$$

and that the lattice join and meet are given, in terms of the operation " $\rightarrow$ " by the formulae in the sumary. Conversely in any orthomodular lattice one can define an operation " $\rightarrow$ " by equation (1.1) and this operation has the properties II through to I4.

Finally I5 is equivalent to

$$
(y \wedge x) \vee N x=(N y \wedge N x) \vee y .
$$

This is clearly true in a Boolean algebra. Conversely if (1.2) holds in the orthomodular lattice $L$ one deduces easily

$$
x \wedge y=0 \text { if and only if } x \leq N y \text {. }
$$

From this one deduces that complementation in $L$ is unique, and hence, Birkhoff [2] (Theorem 17, p. 44), that $L$ is a Boolean algebra.

\section{Interpretation of the operations}

The interest of the results established above comes about because they indicate that one could proceed axiomatically and introduce the logic of quantum mechanics as an implication algebra, that is as a logic which admits an operation of implication with the properties Il through to I4. 
Conversely a "quantum logic" which does have the structure of an orthomodular lattice is a logic admitting such an operation of material implication. Boolean logics were treated as implication algebras in Abbot and Kleindorfer [1].

In Finch [5] it was shown that if one has a binary operation "o" on a logic $L$ such that

$$
\begin{aligned}
& \text { C1. I } \circ x=x, \\
& \text { C2. if } x \leq y \text { then } x \circ z \leq y \circ z, \\
& \text { C3. if } x \leq y \text { then }(z \circ y) \circ x=z \circ x, \\
& \text { C4. }\{N(x \circ y)\} \circ y \leq N x,
\end{aligned}
$$

then $L$ is an orthomodular lattice and

$$
x \circ y=(x \vee N y) \wedge y \text {. }
$$

In fact $\mathrm{Cl}$ to $\mathrm{CH}$ are equivalent to Il to I4; to see this one need only observe that

$$
x \circ y=N(y \rightarrow N x)
$$

and

$$
x \rightarrow y=N(N y \circ x) .
$$

Similarly I5 is equivalent to

$$
x \circ y=y \circ x \text {. }
$$

In Finch [5] it was suggested that the operation "o" could be interpreted as an operation of logical conjunction. This suggestion seems to run counter to the usual interpretation of the lattice operations in quantum logic, where it is customary to interpret the lattice meet as logical conjunction. It was pointed out in Finch [5] however, that there is a certain plausibility in the properties $\mathrm{Cl}$ through to $\mathrm{Cl}$ when "o" is interpreted as an operation of logical conjunction. There is, perhaps, even more plausibility in the properties Il through to I4, and their consequences, when " $\rightarrow$ " is interpreted as material implication. For instance one has

PROPOSITION 1. In an orthomoduzar lattice $L$

$$
(x \rightarrow y)=1 \text { if and only if } x \leq y \text {. }
$$


Proof.

$$
\begin{aligned}
(x \rightarrow y)=1 & \Leftrightarrow N y \circ x=0 \\
& \Leftrightarrow(N y \vee N x) \wedge x=0 \\
& \Leftrightarrow N y \vee N x=N x \\
& \Leftrightarrow x \leq y .
\end{aligned}
$$

REMARK. It is perhaps worthwhile observing that if one interprets lattice meet as logical conjunction and, by analogy with Boolean logic, introduces material implication by the equation

$$
x+y=N(N y \wedge x)
$$

then one would have $(x \rightarrow y)=1$ if and only if $N y \wedge x=0$. In this case one would not be able to relate "material implication" to "logical implication" in the way of the proposition, except when the logic is a Boolean logic.

As a further consequence of interpreting " $\rightarrow$ " as implication and "o" as conjunction we note an interesting analogy with Brouwerian lattices. We recall that a Brouwerian lattice is a lattice $L$ in which, for any elements $a$ and $b$, the set of all elements $x$ in $L$ such that $a \wedge x \leq b$ has a greatest element $b: a$. As noted in Birkhoff [2] a Brouwerian logic is a Brouwerian lattice with $b: a$ relabelled as $a \rightarrow b$, the operation of implication in the logic. By way of analogy we have

PROPOSITION 2. In an orthomodular lattice

$$
x \leq(y+z) \text { if and only if } x \circ y \leq z \text {. }
$$

Proof. We note firstly that

$$
(y \rightarrow z) \circ y=\{(z \wedge y) \vee N y\} \wedge y=z \wedge y .
$$

Thus

$$
x \leq(y \rightarrow z) \Rightarrow x \circ y \leq(y \rightarrow z) \circ y \leq z \text {. }
$$

Conversely

$$
\begin{aligned}
x \circ y \leq z & \Rightarrow(x \vee N y) \wedge y \leq z \wedge y \\
& \Rightarrow x \vee N y \leq(z \wedge y) \vee N y \\
& \Rightarrow x \leq x \vee N y \leq y \rightarrow z .
\end{aligned}
$$


One of the difficulties in the interpretation of the operation "o" as conjunction is its non-comutativity. For this reason intuition is not a good guide in deciding, in a particular context, whether or not the confunction of $x$ and $y$ should be $x \circ y$ or $y \circ x$. To some extent the preceding results are a help because their analogue with $x \circ y$ replaced by $y \circ x$ lead to a Boolean algebra. To see this we replace $x \circ y$ by $y \circ x$ in Proposition 2 and prove

PROPOSITION 3. If $L$ is an orthomodular lattice in which for any two elements $y$ and $z$ in $L$ there exists an element $z: y$ of $L$ such that

$$
x \leq z: y \text { if and only if } y \circ x \leq z \text {, }
$$

then $L$ is a Boolean algebra.

Proof. Since $N y \circ x \leq x$ one has $x \leq x$ : Ny. Similarly since Ny $\circ y=0$ one has also $y \leq x: N y$. Thus

$$
x \vee y \leq x: N y \text {. }
$$

Since $N y \circ 1=N y$ we deduce

$$
x \vee y=1 \Rightarrow N y \leq x .
$$

Thus

$$
\begin{aligned}
x \wedge y=0 & \Rightarrow N x \vee N y=1 \\
& \Rightarrow x \leq N y .
\end{aligned}
$$

It follows that complementation in the orthomodular lattice $L$ is unique, that is $L$ is a Boolean algebra.

\section{References}

[1] J.C. Abbot and P.R. Kleindorfer, "A new characterisation of Boolean algebras", Amer. Math. Monthly 68 (1961), 697-698.

[2] Garrett Birkhoff, Lattice theory (Colloquium Publ. 25, Amer. Math. Soc., Providence, 3rd ed., 1967).

[3] P.D. Finch, "On the structure of quantum logic", J. Symbolic Logic 34 (1969), 275-282. 
[4] P.D. Finch, "Sasaki projections on orthocomplemented posets", Bull. Austral. Math. Soc. 1 (1969), 319-324.

[5] P.D. Finch, "On the lattice structure of quantum logic", BulZ. Austral. Math. Soc. 1 (1969), 333-340.

Monash University,

Clayton, Victoria. 